\title{
Ehlers-Danlos Syndrome, Hypermobility Type: A Characterization of the Patients' Lived Experience
}

\author{
Brittney Murray, ${ }^{1 *}$ Beverly M. Yashar, ${ }^{2}$ Wendy R. Uhlmann, ${ }^{2,3}$ Daniel J. Clauw, ${ }^{4}$ \\ and Elizabeth M. Petty ${ }^{5}$
}

${ }^{1}$ Division of Cardiology, Johns Hopkins Hospital, Baltimore, Maryland

${ }^{2}$ Department of Human Genetics, University of Michigan Medical School, Ann Arbor, Michigan

${ }^{3}$ Department of Internal Medicine, University of Michigan, Ann Arbor, Michigan

${ }^{4}$ Departments of Anesthesiology, Medicine (Rheumatology) and Psychiatry, University of Michigan Chronic Pain and Fatigue Research, Ann Arbor, Michigan

${ }^{5}$ Department of Pediatrics, University of Wisconsin School of Medicine and Public Health, Madison, Wisconsin

Manuscript Received: 27 July 2012; Manuscript Accepted: 16 September 2013

Hypermobility type Ehlers-Danlos syndrome (EDS-HT) is an inherited connective tissue disorder clinically diagnosed by the presence of significant joint hypermobility and associated skin manifestations. This article presents a large-scale study that reports the lived experience of EDS-HT patients, the broad range of symptoms that individuals with EDS-HT experience, and the impact these symptoms have on daily functioning. A 237-item online survey, including validated questions regarding pain and depression, was developed. Four hundred sixty-six (466) adults ( $90 \%$ female, $52 \%$ college or higher degree) with a self-reported diagnosis of EDS-HT made in a clinic or hospital were included. The most frequently reported symptoms were joint pain (99\%), hypermobility (99\%), and limb pain (91\%). They also reported a high frequency of other conditions including chronic fatigue (82\%), anxiety (73\%), depression (69\%), and fibromyalgia (42\%). Forty-six percent of respondents reported constant pain often described as aching and tiring/exhausting. Despite multiple interventions and therapies, many individuals (53\%) indicated that their diagnosis negatively affected their ability to work or attend school. Our results show that individuals with EDS-HT can experience a wide array of symptoms and co-morbid conditions. The degree of constant pain and disability experienced by the majority of EDS-HT respondents is striking and illustrates the impact this disorder has on quality of life as well as the clinical challenges inherent in managing this complex connective tissue disorder. $\odot 2013$ Wiley Periodicals, Inc.

Key words: Ehlers-Danlos syndrome; hypermobility; disability; chronic pain; joint laxity; Type III; connective tissue disorders; lived experience

\section{INTRODUCTION}

Ehlers-Danlos syndrome (EDS) is a group of highly variable heritable connective tissue disorders characterized by a wide range of clinical manifestations that most often include skin manifesta-
How to Cite this Article:

Murray B, Yashar BM, Uhlmann WR, Clauw DJ, Petty EM. 2013. Ehlers-Danlos syndrome, hypermobility type: A characterization of the patients' lived experience.

Am J Med Genet Part A 161A:2981-2988.

tions and joint hypermobility. Some forms of EDS (i.e., classic, vascular) are caused by defects in the synthesis, processing, secretion, or stability of fibrillar collagen molecules; which is a major structural component of connective tissue, skin, vasculature, tendons, and joint ligaments. The variety of symptoms, in the different forms of EDS, overlap in the clinical presentation. Classic type EDS, associated with mutations in COL5A1 and COL5A2, involves mainly skin hyperextensibility, abnormal wound healing, and some hyperextensibility. Vascular type EDS, considered the most life threatening, involves severe fragility of connective tissues with arterial and gastrointestinal ruptures, and complications of surgical interventions. Vascular type EDS is mostly commonly associated with mutations in COL3A1 [Germain and Herrera-Guzman, 2004; Fernandes and Schwartz, 2008]. However, one of the most prevalent, but often under-diagnosed, forms of EDS is hypermobility

The authors have no conflict of interest to declare.

Grant sponsor: Rackham Graduate School at the University of Michigan. ${ }^{*}$ Correspondence to:

Brittney Murray, Division of Cardiology, Johns Hopkins Hospital, 600 N. Wolfe St., Blalock 545, Baltimore, MD 21287.

E-mail: bmurray@jhmi.edu

Article first published online in Wiley Online Library

(wileyonlinelibrary.com): 6 November 2013

DOI 10.1002/ajmg.a.36293 
type EDS (EDS-HT) for which the molecular basis remains largely unknown [Tinkle, 2008; Tinkle et al., 2009].

EDS-HT is associated with chronic, frequently debilitating, musculoskeletal pain, and ongoing joint hypermobility, often resulting in severe arthritis and physical limitations [Castori et al., 2012]. There are also anecdotal reports of a high incidence of co-morbid conditions including fatigue, fibromyalgia, migraines, and irritable bowel syndrome [Sacheti et al., 1997; Adib et al., 2005; Tinkle, 2008; Tinkle et al., 2009; Castori et al., 2010; Rombaut et al., 2010]. The current diagnostic criteria to establish a clinical diagnosis of EDS-HT in adults includes a hypermobility score of 5 or greater on the 9-point Beighton scale, soft skin with normal or minimally increased extensibility and absence of fragility or other significant skin or soft tissue abnormalities. The diagnosis may also be supported by the occurrence of minor criteria that include joint dislocations, easy bruising, functional bowel disorders, chronic limb/joint pain, or a positive family history [Beighton et al., 1998]. More recent work has highlighted the increase in additional health complaints including musculoskeletal, pseudoneurological, and gastrointestinal symptoms [Maeland et al., 2011].

Though there are diagnostic criteria, EDS-HT continues to be underdiagnosed. The condition is difficult to recognize as individuals may present with chronic pain without other major symptoms other than increased joint hypermobility and no other objective findings on imaging or laboratory studies. Studies have found that only $10 \%$ of physicians referring EDS-HT patients to rheumatology clinics realized that their joint hypermobility was the underlying cause of their patients' pain [Adib et al., 2005]. It has been proposed that EDS-HT may be the occult underlying diagnosis for many chronic somatic pain syndromes [Castori et al., 2013].

This chronic pain can lead to considerable psychological distress and reduced quality of life. Current treatments modalities for EDSHT are of limited benefit and are restricted to symptomatic management, often with pain medications and physical therapy [Sacheti et al., 1997; Rombaut et al., 2011; Castori et al., 2012]. Treatment is generally ineffective or suboptimal and individuals with a diagnosis of EDS-HT often experience depression and a lower quality of life that limit their lifestyles, as well as affecting their psychological well being [Ainsworth and Aulicino, 1993; Tinkle, 2008]. Baeza-Velasco et al. [2011] found that chronic pain in these individuals with a diagnosis of EDS-HT is often associated with depression and anxiety that is amplified by a lack of recognition and knowledge of the syndrome by managing clinicians. There is also a body of literature that suggests that EDS-HT disease-related symptoms severely affected individuals' quality of life. Other problematic issues emphasized in this population include stigmatization, altered life plans, and constant fear of the pain and its progression [Berglund et al., 2000]. New recommendations published by Castori et al. [2012] suggest extensive lifestyle counseling including a multidisciplinary team.

To date, there have been limited studies that have focused on comprehensively describing the experience of living with EDS-HT. Castori et al. [2010] surveyed 21 EDS-HT patients seen in a genetics clinic, Castori et al. described the musculoskeletal, gastrointestinal, neurological, cardiovascular, urogynecological, and ear-nose- throat symptoms they experienced but failed to explore their psychological state. While other studies by Voermans et al. $[2009,2010]$ have focused on specific types of symptoms associated with EDS-HT, such as fatigue and pain, they did not describe the full range of clinical symptoms experienced by their study population. In a sample of 273 individuals with EDS (162 of which with EDSHT) they looked specifically at the impact of pain and concluded that pain is common in severe EDS. Recently, Remvig et al. [2011] described the need for a broader appreciation of the full scale of multi-system involvement in EDS-HT. Maeland et al. [2011] have also reported that up to $98 \%$ of individuals with EDS-HT report substantial complaints in the last 30 days.

The complex health issues that surround a diagnosis of EDS-HT demand a comprehensive evaluation/catalogue of both the clinical and lived experience to better aid the practitioner in managing these patients. Our study objective was to comprehensively describe the experience of living with a diagnosis of EDS-HT from the patient perspective. We surveyed a large number of individuals with a diagnosis of EDS-HT to describe symptoms and pain experienced by individuals with this condition.

\section{METHODS}

\section{Recruitment}

This study was conducted from September 2009 to April 2010. Patients with a clinical diagnosis of EDS-HT were recruited from two sources. The first recruitment arm was through two support groups: the Ehlers-Danlos National Foundation (EDNF) and the local Ehlers-Danlos Support Group (AnnArborEDS@gmail.com). Following approval of the study by their boards of directors, the study was announced on each group's website with a direct link to a web-based survey (content described below). The study announcement specified the inclusion criteria that individuals must be over 18 years of age and by their self-report to have a clinical diagnosis of EDS-HT made by a physician. The second recruitment arm was through the University of Michigan Medical Genetics Clinic. Adult patients seen in the past 5 years with a confirmed clinical diagnosis EDS-HT were sent a letter of recruitment and provided with the address to the web-based survey. The survey was accessible for a 2-month period. This study was approved by the University of Michigan Internal Review Board (HUM00031584).

\section{Study Instrument}

A 237-item survey instrument was developed by the research team that ascertained the respondents' self-reported information about (1) demographics, (2) mechanism for EDS-HT diagnosis, (3) symptom/comorbid conditions, (4) pain and depression, (5) impact on of diagnosis on work and education, and (6) sources of support (Table I). The survey instrument contained both validated measures (71 items) and those that were developed specifically for this study. As the goal of this study was to explore the perceived impact of an EDS-HT diagnosis on an individual's life, we gathered this information by participant self-report. The survey questions that elicited participant reports of symptoms/comorbid conditions and treatments were developed from an analysis of the current 


\section{TABLE I. Survey Instrument Components}

\section{Instrument}

Symptom catalog

Treatment catalog

Melzack [1987]

McGill Pain Questionnaire

[short form]

The Center for Epidemiologic

Studies Depression

Scale (CES-D)
Research Team

Authors

Research Team

Radloff [1977]

\section{Description}

Systematic checklist of current symptoms related to disease, duration, frequency, age of onset, and priority in terms of a ranking of the symptoms that have the most effect

Self-report checklist of current and past caregivers, treatments tried, duration, whether tried in the past or currently, relative efficacy

Scale that measures intensity and quality of pain by requesting participants to rate pain through 15 verbal descriptors on a $0-3$ rating scale. Widely used for assessment of clinical pain.

Scale that asks how individuals have felt in the last week; a score of greater than 16 on this test indicates "probable depression."

\section{Focus as applied to this research}

Create a comprehensive description of symptoms experienced by individuals with EDS-HT.

Explore the management options utilized by individuals with EDS-HT.

Describes the level of pain experienced by EDS-HT patients.

Assess the psychological impact of EDSHT. This scale was only completed when individuals responded "Yes" to having ever experienced depression. literature by the research composed of physicians and genetic counselors who regularly work with a individuals with a diagnosis of EDS-HT. An integral component of the survey development included consultation with the leader of a local EDS support group. The survey instrument was converted into an online form and administered through Qualtrics survey software.

\section{Statistical Analysis}

Data were analyzed using PASW (Predictive Analytic Software) Statistics 17. Responses were excluded if the respondent did not reach the end of the survey, and/or if they reported their diagnosis was made anywhere other than in a clinic/hospital. Analysis was performed using descriptive statistics: frequencies and means, as well as Pearson chi-square tests and correlations. Reported frequencies and means were determined to be significant using a onesided $t$-test. Values were considered significant when analysis by $t$-test determined that the value in this population was significantly different than expected by chance alone.

\section{RESULTS}

\section{Demographics of the Study Population}

Out of the initial 617 respondents (580 from the support group websites, and 37 identified from the University of Michigan Medical Genetics Clinic), the final data analysis group consisted of 466 individuals. Respondents were excluded if they did not reach the end of the survey (44), or if they did not report that their diagnosis of EDS-HT had been made in a clinic or hospital (107). As this was a web-based survey, the response rate is unknown, however of those identified from the Medical Genetics Clinic, there was a $41 \%$ response rate from this group (37/91), but this was only $6 \%$ of the overall response. Demographics of the study population are listed in Table II. The population was 95\% Caucasian and $89 \%$ had completed some college or higher; notably, $22 \%$ had Master's or

\section{TABLE II. Demographics of the Study Population $[n=466$ ]}

Sex

Female

Male

89.9

Race

White/Caucasian $\quad 95.3$

Hispanic $\quad 2.1$

Asian $\quad 1.5$

Black/African American $\quad 0.9$

Other

Education

Some high school

1.9

Completed high school $\quad 8.8$

Some college

Bachelor's degree

36.7

Graduate level

20.2

Doctorate

25.8

6.2

Geographic location

Midwest

25.5

Northeast

17.8

Southeast 12.7

Southwest $\quad 8.8$

Northwest $\quad 7.5$

Central Southern $\quad 5.4$

Country outside the USA 21.7

Doctorate degrees. Sixty-nine percent of respondents resided within 30 miles of a major academic healthcare facility.

\section{Clinical Symptoms and Related Diagnoses}

The majority of participants reported being diagnosed in either a genetics or rheumatology clinic (Table III). Fifty-six percent of participants had been seen in a genetics clinic at some point in the 


\begin{tabular}{|c|c|c|}
\hline Clinic setting & $\begin{array}{c}\text { Diagnosis } \\
\text { established [\%] }\end{array}$ & $\begin{array}{c}\text { Primary } \\
\text { caregiver [\%] }\end{array}$ \\
\hline Primary care & 5.8 & 41.5 \\
\hline Genetics & 28.6 & 6.2 \\
\hline Rheumatology & 1.5 & 18.9 \\
\hline Orthopedics & 7.3 & 6.9 \\
\hline Physical medicine & 1.5 & 4.9 \\
\hline Pediatrics & 1.3 & 0.9 \\
\hline Pain clinic & 0.4 & 6.0 \\
\hline Other & 3.9 & 9.0 \\
\hline
\end{tabular}

past. Forty-two percent of participants reported that the main physician who manages their diagnosis was their primary care physician and 19 percent reported that a rheumatologist provides primary clinical management of their disease.

The significance of a diagnosis of EDS-HT on the health of respondents was catalogued by assessing the type of symptoms, frequency, age of onset, and reported impact on participants' lives. Respondents reported experiencing a broad range of symptoms and diagnoses that impacted the following categories: their joints (99\%), cardiovascular system (96\%), gastrointestinal system (96\%), skin (95\%), neurological/psychological manifestations (88\%), and genitourinary system (67\%) (Fig. 1). Joint symptoms were the most common category reported, which is consistent with the EDS-HT clinical diagnostic criteria. Among 39 possible symptoms or diagnoses surveyed in the study, the most commonly reported symptoms were joint pain, hypermobility, limb pain, and joint subluxations. Participants also frequently reported dizziness and chronic fatigue. In Figure 1, starred symptoms represent those that were reported by a significant number of respondents rather than expected by chance $(P<<0.001)$.

Thirty-three percent of participants reported experiencing between 15 and 25 symptoms and/or clinical diagnoses. The majority of respondents' reported that their symptoms presented before the age of 30. Hypermobility and skin findings were noted to present in childhood (under age 18) for a majority of individuals. A significant proportion of participants reported that joint pain, dislocations, and subluxations began under the age of 18 (79\% of respondents). For respondents with depression, anxiety, and chronic fatigue age of onset range between 18 and 30 years of age.

A significant number of respondents reported experiencing depression (69\%) and anxiety (73\%). Respondents who indicated that they had experienced depression were then prompted to complete the Center for Epidemiologic Studies Depression Scale (CES-D). Of those that filled out the CES-D (321/466), 98\% had elevated scores; a score of over 16 is reported as an increased susceptibility for depression (range of scores 1-46) [Radloff, 1977]. Figure 2 shows the impact that participants reported these symptoms have on their lives.

\section{Pain and Depression}

Respondents reported chronic pain and a large proportion (67\%) reported that the pain as constant, as opposed to intermittent (experienced several times a day) (22\%), multiple times per week $(6.5 \%)$, once per week $(1.6 \%)$, or once per month (1.6\%). Only $0.6 \%$ of the study respondents reported they did not experience pain on a regular basis. Those individuals who reported a higher levels of pain were also more likely to report experiencing a significantly higher number of symptoms $(P<0.05)$. The most commonly reported pain types reported were tiring/exhausting pain (93\%) and aching (80\%) (Fig. 3). Participants with tiring and exhausting pain also reported symptoms of chronic fatigue (92\%), depression (78\%), and fibromyalgia (56\%). For respondents with tiring and exhausting pain, $42 \%$ reported taking an anti-depressant medication.

\section{Effect of EDS-HT on Work and School}

Individuals were asked to describe the effect that their diagnosis had on their employment or education. Two hundred fifty-one respondents (55\%) were currently employed, $24 \%$ were only working part time due to having EDS-HT. Of all of those working, $52 \%$ had to change roles/take on less responsibility because of their diagnosis. Sixty-six percent reported that their employer knew of their diagnosis. Fifty-four percent of the respondents who were not working (54/466) indicated that limitations due to their diagnosis of EDSHT was the reason. There were 119 respondents who were currently students, and of those, and 21 of $119(18 \%)$ indicated that they were not able to enroll in a fulltime educational program as a consequence of their diagnosis. There were an additional 38 respondents who indicated they were not enrolled in school due to having a diagnosis of EDS-HT. Of the responses regarding performances, $49 / 60(82 \%)$ strongly agreed or agreed that having this diagnosis had affected their performance at work or school and 33 (55\%) indicated that special accommodations were needed.

\section{Sources of Support}

A variety of questions were asked inquiring about the support resources utilized by this population. Fifty-seven percent reported that they regularly read EDS literature, websites, or publications and $11 \%$ reported currently attending support group meetings. Participants were asked about sources of support: $62 \%$ felt supported by their family, $52 \%$ by friends, $25 \%$ by religious institutions, and $24 \%$ by their employer.

\section{DISCUSSION}

In this large-scale study of the lived experience of EDS-HT, participants report a number of symptoms and comorbid diagnosis outside of joint hypermobility alone. Important findings in these results are the shear number of symptoms these patients experience and the pain that accompanies them. This pain and depression that has a destructive effect on quality of life in these individuals, which participants report has ripple effects on their work and/or school success. While a patient-report based survey has limitations, the authors felt understanding the lived experience of the individual would be critical in advising better management in this population. These results were consistent with previous studies in that a multidisciplinary team approach may be most effective in 


\section{EDS-HT patients' self-reported symptoms and diagnoses}

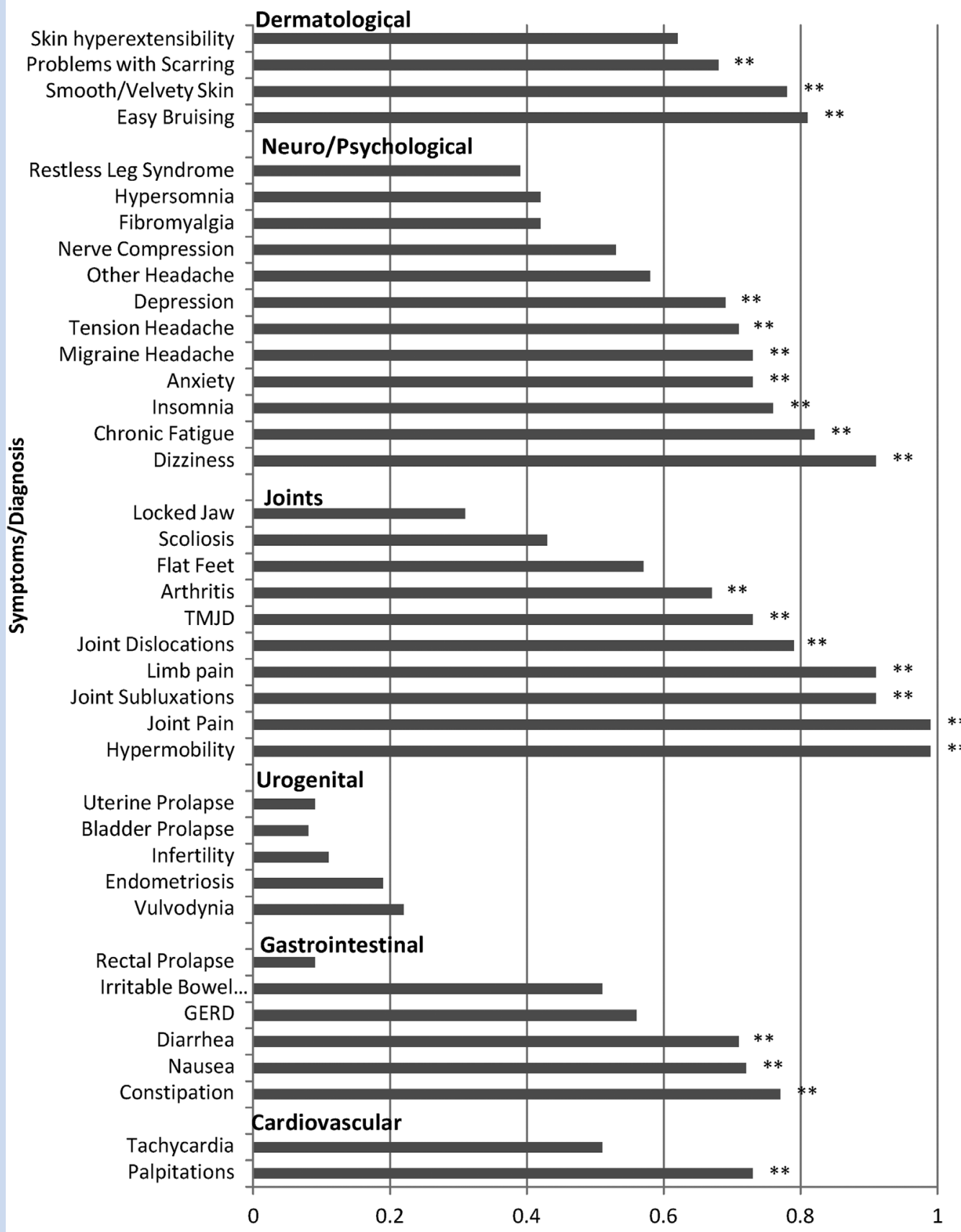

Percentage of participants self-reporting this symptom/diagnosis

FIG. 1. EDS-HT symptoms and frequency in study population. Participants were asked if they had ever experienced any of the listed 39 symptoms, frequencies of which are displayed in the figure [\%]. Those starred were experienced by a significant proportion of the respondent population as described $[P<<0.001)$. Symptoms are grouped by type: cardiovascular, gastrointestinal, genitourinary, joints, nervous system, psychological, or skin findings.

managing these patients [Hakim and Grahame, 2003; Grahame, 2009; Castori et al., 2012].

Participants reported a broad range of symptoms from skin scarring and hyperextensibility to heart palpitations, gastrointes- tinal distress, and significant psychological concerns in addition to the expected hypermobility symptoms. A large percentage of participants reported between 15 and 25 symptoms and/or related clinical diagnoses, supporting the multisystemic nature 


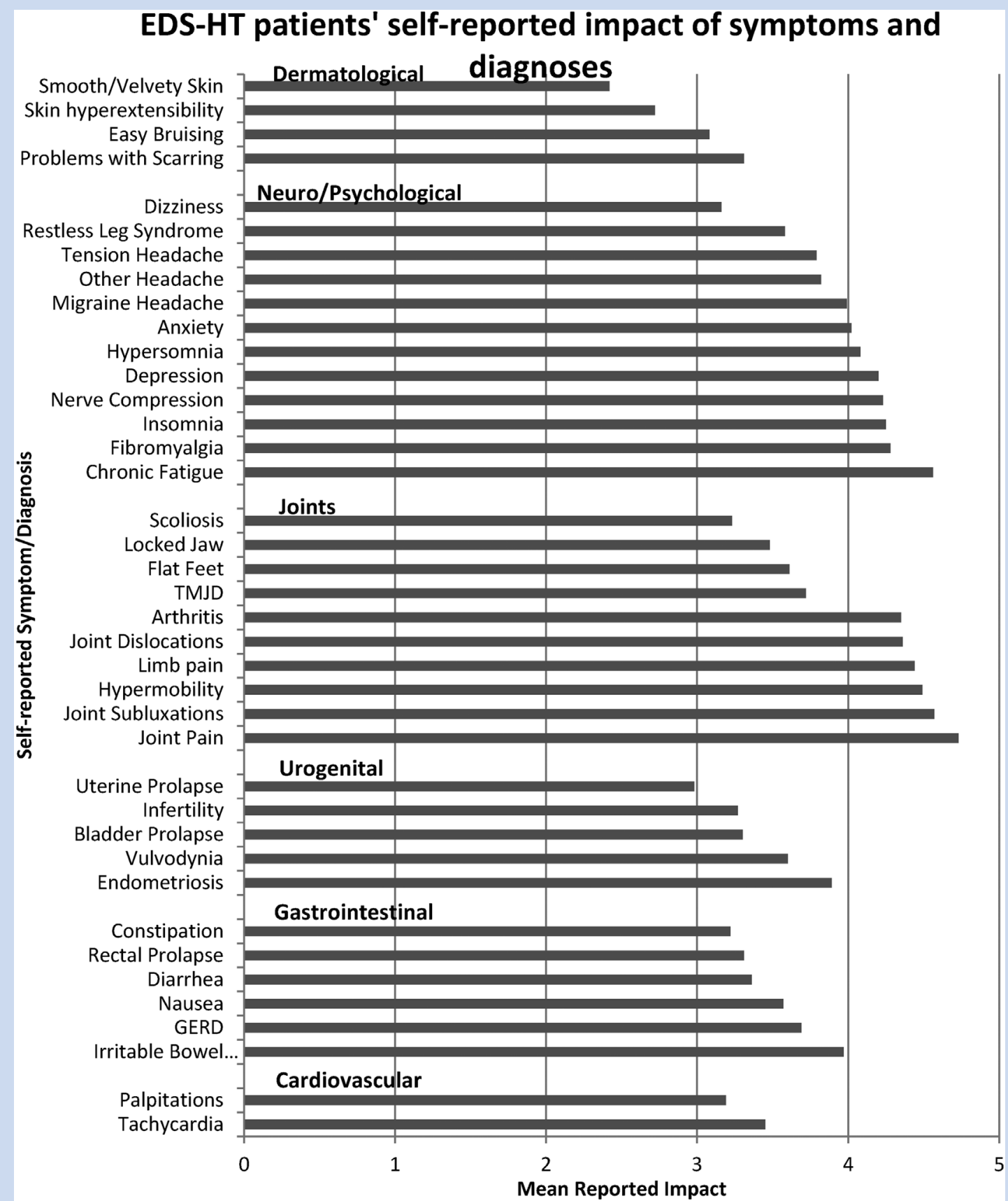

FIG. 2. Impact of EDS-HT symptoms. The mean impact that respondents reported each symptom had on their quality of life is shown. Participants were asked to rank each symptom on a 1-5 Likert scale, (1 = "Strongly Disagreed" that the symptom had a significant impact, and $\mathbf{5}=$ "Strongly Agreed" that the symptom impacted their quality of life].

of this condition. To the patients, this diagnosis is far from benign, and mislabeling EDS-HT as benign hypermobility is not only misleading to patients, and providers, but it significantly diminishes validation of the lived experience that affected individuals report. Lack of validation may contribute to problems with patient-health care provider interactions and, importantly, result in suboptimal clinical management [Baeza-Velasco et al., 2011].
It has been well documented that one of the most pressing frustrations of individuals with chronic pain is the fact that there is a lack of objective findings to support their subjective feelings [Berglund et al., 2010; Dow et al., 2012]. Beyond the physical impact of the disease, our data further indicate that a diagnosis of EDS-HT impacts quality of life and can significantly impact psychological well-being and the ability to function within the normal adult environments such as work, school, and family. Our results are 


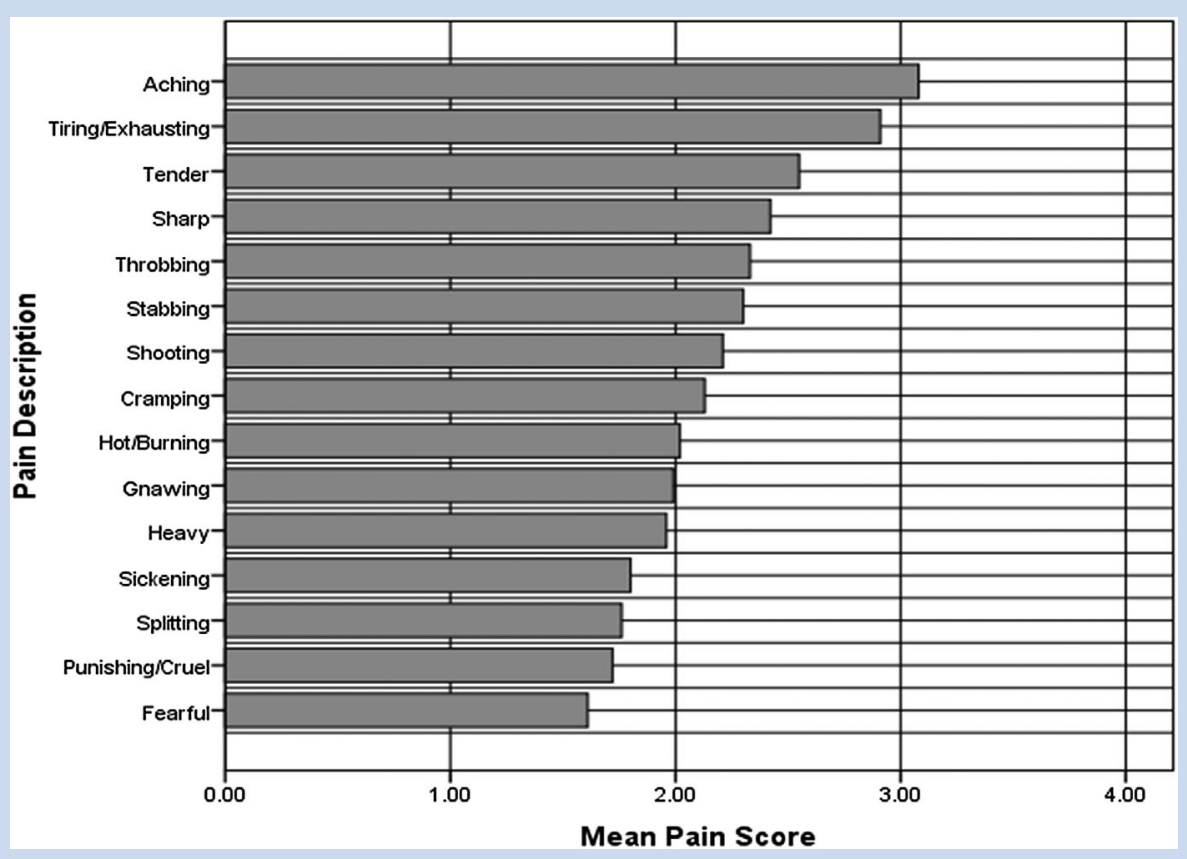

FIG. 3. Type of pain experienced by EDS-HT study population. The graph shows the mean pain score reported by participants on the McGill pain questionnaire [Melzack, 1987]. Participants ranked the pain they felt as 1 ="No pain," 2 ="Mild pain," 3 ="Moderate pain," and 4 ="Worst possible pain, or severe." The mean pain score for each type of pain was calculated for all participants and is shown in the figure.

consistent with those in smaller studies of EDS-HT patients which have found that their health related quality of life is significantly lower on all sections of a health related quality of life scale than healthy gender- and age-matched controls [Rombaut et al., 2010].

This lowered quality of life can also be compounded by the fact that a diagnosis of EDS-HT may exist as an "invisible disability." As described by Davis [2005], invisible disabilities occur as "individuals with conditions, illnesses, and structural or biomechanical anomalies that are life limiting but not readily discernible to others." Davis highlights that understanding the lived experience of the individual is crucial to both understanding the disability and also in diagnosing and characterizing the disorder. Our results provide key information that will increase the visibility of the life limiting aspects of an EDS-HT diagnosis. They highlight large gaps in the current clinical picture of EDS-HT.

The results of this study are limited by our recruitment methods and self-reported nature of the clinical diagnoses. As a survey-based study it is not possible to validate symptoms or clinical diagnoses reported by the respondents, however, this method enables us to develop a comprehensive description of the experiences of those who are living with a diagnosis of EDS-HT that can be used to health care providers' perspective. Diagnoses were by self-report and no medical records were reviewed for confirmation. Most participants, as noted above, reported symptoms in line with the diagnostic criteria. The recruitment methods may have created a bias as individuals who use EDS-HT support group websites (94\% of our respondents) may have more symptoms and a higher level of pain than those who do not access support group resources. In addition, those contacted directly from the clinic may have also used the support group resources, and as they have been seen in a tertiary genetics center, may have different results. Response from the clinic population, however, was not large enough to compare these two groups. There were significantly more female respondents ( $89 \%$ ) than males; however, this is consistent with the reported population of individuals who carry a diagnosis of EDSHT [Voermans et al., 2009]. While parts of the survey use validated survey instruments were used, other survey questions were developed by the research team and were not validated. Questions were subject to participants' interpretation of the study questions. No definitions of terms were provided. The length and internet-basis of the instrument may also have created biases.

This is a large-scale study that provides a comprehensive summary of the self-reported symptoms, clinical diagnoses, and life experiences of individuals who have a diagnosis of EDS-HT. We explored the lived experience of individuals with a diagnosis of EDS-HT: their medical symptoms, perspectives on their disease, experiences with pain and resulting disability, and support systems to gain a deeper understanding of the morbidity that individuals' experience. As alluded to in previous smaller studies, the data suggest that there is more complexity to the clinical manifestations of EDS-HT than joint hypermobility and mild skin manifestations [Castori et al., 2010; 2012]. Documentation of the broad array of symptoms/clinical diagnoses and effects on quality of life reported by this population in this study has important implications for health care providers caring for this population. Data from this and other studies strongly support the need for better recognition, understanding, and management of EDS-HT as a multisystemic, often disabling condition [Hakim and Grahame, 2003; Grahame, 
2009; Castori et al., 2012]. In addition, due to the multiple systems reported as affected by these individuals, this work strongly supports recent studies that a multidisciplinary approach to management is necessary [Castori et al., 2012].

\section{ACKNOWLEDGMENTS}

The authors would like to thank the support and guidance of the Ehlers-Danlos National Foundation (EDNF) and the Ehlers-Danlos Support Group and their participants. Without their support and cooperation, this research would have had limited reach. The authors would also like to thank Ms. Katherine Kerschbaum for her input, expertise, and guidance in the development and execution of this study. This study was also supported by a grant from the Rackham Graduate School at the University of Michigan.

\section{REFERENCES}

Adib N, Davies K, Grahame R, Woo P, Murray KJ. 2005. Joint hypermobility syndrome in childhood. A not so benign multisystem disorder? Rheumatology 44:744-750.

Ainsworth SR, Aulicino PL. 1993. A survey of patients with Ehlers-Danlos syndrome. Clin Orthop Relat Res 286:250-256.

Baeza-Velasco, Gely-Nargeot MC, Bulbena Vilarrasa A, Bravo JF, 2011. Joint hypermobility syndrome: Problems that require psychological intervention. Rhematol Int 31:1131-1136.

Beighton P, De Paepe A, Steinmann B, Tsipouras P, Wenstrup RJ. 1998. Ehlers Danlos syndrome: Revised nosology, Villefrache 1997, 1998. Ehlers-Danlos National Foundation (USA) and Ehlers-Danlos Support Group (UK). Am J Med Genet 77:31-37.

Berglund B, Nordstrom G, Lutzen K. 2000. Living a restricted life with Ehlers Danlos Syndrome. Int J Nurs Stud 37:111-118.

Berglund B, Anne-Cathrine M, Randers I. 2010. Dignity not fully upheld when seeking health care: Experiences expressed by individuals suffering from Ehlers-Danlos syndrome. Disabil Rehabil 32:1-7.

Castori M, Camerota F, Celletti C, Danese C, Santilli V, Saraceni VM, Grammatico P. 2010. Natural history and manifestations of the hypermobility type Ehlers-Danlos syndrome: A pilot study on 21 patients. Am J Med Genet Part A 152A:556-564.

Castori M, Celletti C, Camerota F. 2013. Ehlers-Danlos syndrome hypermobility type: A possible unifying concept for various functional somatic syndromes. Rheumatol Int. 33:819-821.

Castori M, Morlino S, Celletti C, Celli M, Morrone A, Colombi M, Camerota F, Grammatico P. 2012. Management of pain and fatigue in the joint hypermobility syndrome (a.k.a. Ehlers-Danlos syndrome, hypermobility type): Principles and proposal for a multidisciplinary approach. Am J Med Genet Part A 158A:2055-2070.

Davis NA. 2005. Invisible disability. Ethics 116:153-213.
Dow CM, Roche PA, Ziebland S. 2012. Talk of frustration in the narratives of people with chronic pain. Chronic Illn 8:176-191.

Fernandes NF, Schwartz RA. 2008. A "hyperextensive” review of EhlersDanlos syndrome. Cutis 82:242-248.

Germain DP, Herrera-Guzman Y. 2004. Vascular Ehlers-Danlos syndrome. Ann Genet 47:1-9.

Grahame R. 2009. Joint hypermobility syndrome pain. Curr Pain Headache Rep 13:427-433.

Hakim A, Grahame R. 2003. Joint hypermobility. Best Pract Res Clin Rheumatol 17:989-1004.

Maeland S, Assmus J, Berglund B. 2011. Subjective health complaints in individuals with Ehlers Danlos syndrome: A questionnaire study. Int J Nurs Stud 48:720-724.

Melzack R. 1987. The short-form McGill Pain Questionnaire. Pain 30:191197.

Radloff LS. 1977. The CES-D scale: A self-report depression scale for research in the general population. Appl Psychol Meas 1:385-401.

Remvig R, Engelbert RH, Berglund B, Bulbena A, Byers PH, Grahame R, Juul-Kristensen B, Lindgren KA, Uitto J, Wekre LL. 2011. Need for a consensus on the methods by which to measure joint mobility and the definition of norms for joint hypermobility that reflect age, gender, and ethnic-dependent variation: Is revision of criteria for joint hypermobility syndrome and Ehler-Danols syndrome hypermobility type indicated? Rheumatology 50:1171-1173.

Rombaut L, Malfait F, Cools A, De Paepe AD, Calders P. 2010. Musculoskeletal complaints, physical activity and health-related quality of life among patients with the Ehlers-Danlos syndrome hypermobility type. Disabil Rehabil 32:1339-1345.

Rombaut L, Malfait F, DeWandele I, Cools A, Thijs Y, DePaepe A, Calders P. 2011. Medication, Surgery, and physiotherapy among patients with hypermobility type of Ehlers-Danlos syndrome. Arch Phys Med and Rehabil 92:1106-1112.

Sacheti A, Szemore J, Bernstein B, Tafas T, Schechter N, Tsipouras P. 1997. Chronic pain is a manifestation of the Ehlers Danlos syndrome. J Pain Symptom Manage 14:88-93.

Tinkle BT. 2008. Issues and management of joint hypermobility: A guide for the Ehlers Danlos syndrome hypermobility type and hypermobility syndrome, 1st edition. Green Fork: Left Paw Press. p 148.

Tinkle BT, Bird HA, Grahame R, Lavallee M, Levy HP, Sillence D. 2009. The lack of clinical distinction between the hypermobility type of Ehlers-Danlos syndrome and the joint hypermobiltiy syndrome (a.k.a. hypermobility syndrome). Am M Med Genet Part A 149A: 2368-2370.

Voermans NC, Knoop H, van de Kamp N, Hamel BC, Bleijenberg G, van Engelen BG. 2009. Fatigue is a frequent and clinically relevant problem in Ehlers-Danlos syndrome. Semin Arthritis Rheum 40:267-274.

Voermans NC, Knoop H, Bleijenberg G, van Engelen BG. 2010. Pain in Ehlers-Danlos syndrome is common, severe, and associated with functional impairment. J Pain Symptom Manage 40:370-378. 